\title{
Effects of Dietary Manganese on Oxidative Damage of the Neurons in Cocks
}

\author{
Xiaofei Liu, Lirong Jing, Lv Xue, Yongqiang Ma, Huanan Guan, Wang Wei \\ College of Food Engineering, Harbin University of Commerce, Harbin 150076, China \\ email: liuxiaofei72@163.com
}

Keywords: Manganese; Oxidative Damage; Neurons; Cocks

\begin{abstract}
To investigate the Manganese(Mn)-induced toxicity on crucial oxidative damage parameters on brain of birds, 50-day-old male Hyline cocks were fed either a commercial diet or a Mn-supplemented diet. The following were determined: restraining ability to $\mathrm{OH} \cdot$, the activities of $\mathrm{Na}+-\mathrm{K}+$-ATPase, $\mathrm{Mg}^{2+}$-ATPase and $\mathrm{Ca}^{2+}$-ATPase, the activities of succinate dehydrogenase (SDH) and Calcineurin (CaN); Exposure to $\mathrm{Mn}$ significantly lowered restraining ability to $\mathrm{OH} \cdot$, the activities of ATP enzymes, activities of SDH and CaN. These findings suggested that Mn exposure resulted in the oxidative damage of cock cerebral tissue.
\end{abstract}

\section{Introduction}

Manganese (Mn) is an essential trace element required for normal development and organism functions. However, exposure to $\mathrm{Mn}$ is usually the result of environmental contamination by waste from human activities. It is a common finding that a wild bird is affected by Mn toxicity. Piscivorous birds are in the upper trophic chain levels of these ecosystems, and as a consequence, they bioaccumulate hydrophobic contaminants [1].

Several reports propose that $\mathrm{Mn}$ neurotoxicity is mainly associated with mitochondrial dysfunction leading to decreased oxidative phosphorylation, increased reactive oxygen species (ROS) generation [2] and as a consequence apoptotic cell death. Among all of the ROS hydroxyl radicals $(\mathrm{OH} \cdot)$ have the highest destructive potential. Heavy metals are important contributors of cell apoptosis. They have high affinity for enzymes and proteins containing thiol groups, which are responsible for normal cellular defense mechanisms. Long term exposure to heavy metals could lead to apoptosis [3]. The aim of this study was to provide a better understanding of the oxidative damage of bird cerebral tissue induced by excessive Mn using the Hyline cock brain as a model.

\section{Materials and methods}

All procedures used in this research were approved by the Institutional Animal Care and Use Committee of Northeast Agricultural University. The method according to Liu et al [4] .

Restraining ability to $\mathrm{OH} \cdot$ in brain was measured spectrophotometrically. The activities of $\mathrm{Na}^{+}-\mathrm{K}^{+}$-ATPase, $\mathrm{Mg}^{2+}$-ATPase, $\mathrm{Ca}^{2+}$-ATPase, and SDH were determined using commercial kits according to the manufacturer's protocol. The SDH and CaN activity was determined using SDH activity assay kits and CaN activity assay kits according to the manufacturer's instructions, respectively. The cleavage of genomic DNA during apoptosis yields single strand breaks (nicks) in high molecular weight DNA. Apoptotic nuclei in tissue sections were identified using TUNEL technique [5]. All statistical analyses were performed using SPSS procedures.

\section{Results}

The results of $\mathrm{OH}$ - were shown in Fig.1. At each dose point of the experiment the variances of the inhibition ability of $\mathrm{OH} \cdot$ in brain of $30 \mathrm{~d}$ was not significant and decreased at 60 and $90 \mathrm{~d}$ gradually. In each dose group the inhibition ability of $\mathrm{OH}^{-}$in the control group increased firstly, then decreased and presented a decreased tendency in the Mn treatment dose group. Notably, the inhibition ability of $\mathrm{OH} \cdot$ in the $\mathrm{M}$ group and $\mathrm{H}$ groups at $90 \mathrm{~d}$ were $16.1 \%$ and $23.7 \%$ lower, 
respectively $(p<0.01)$.

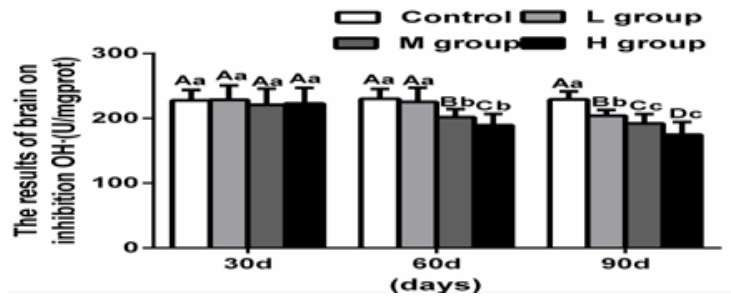

Fig. 1 Effects of Mn poisoning on the inhibition ability of $\mathrm{OH} \cdot$. in brain.

Note: Bars represent the mean \pm standard deviation ( $n=30$ /group). Bars with different uppercase letters are statistically significantly different in the same group. Bars with different lowercase letters are statistically significantly different at the same concentrations of dietary Mn.

The effects of $\mathrm{Mn}$ on the activities of $\mathrm{Na}^{+}-\mathrm{K}^{+}$-ATPase(Fig.2a), $\mathrm{Mg}^{2+}$-ATPase(Fig. 2b) and $\mathrm{Ca}^{2+}$-ATPase(Fig.2c) in were shown in Fig.2. The three ATPase activities at 30 and $60 \mathrm{~d}$ increased firstly and then decreased In addition, they were significantly lower in the brain (30 d, $60 \mathrm{~d}$ and $90 \mathrm{~d}$ ) of the $\mathrm{Mn}$ treatment groups $(p<0.05)$. The current findings indicate that excessive $\mathrm{Mn}$ significantly attenuated the activities of $\mathrm{Na}^{+}-\mathrm{K}^{+}$-ATPase, $\mathrm{Mg}^{2+}$-ATPase, and $\mathrm{Ca}^{2+}$-ATPase.

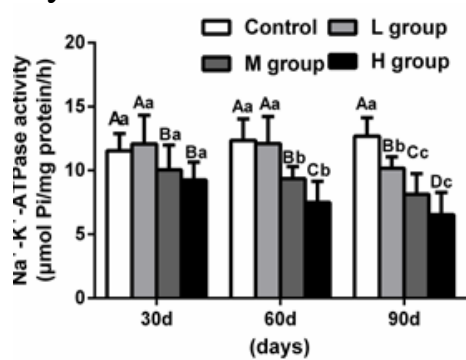

(a) $\mathrm{Na}^{+}-\mathrm{K}^{+}$-ATPase

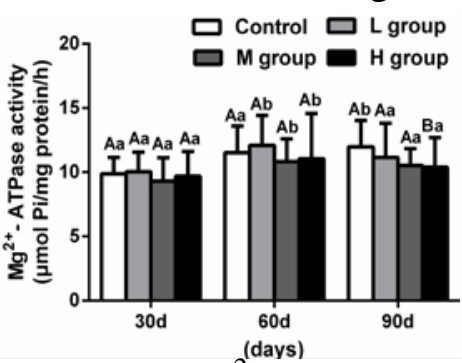

(b) $\mathrm{Mg}^{2+}$-ATPase $\square$ Control $\square$ L group

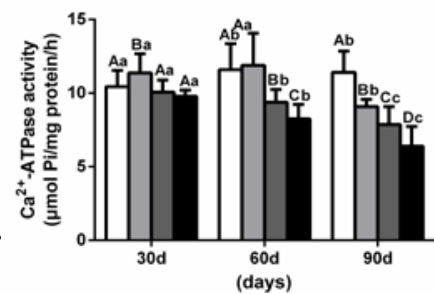

(c) $\mathrm{Ca}^{2+}$-ATPase

Fig.2 Effects of Mn poisoning on the variation of ATP enzymes activity

The results of SDH activity and CaN activity in brain was shown in Fig.3a and Fig.3b. the SDH activity at 60 and $90 \mathrm{~d}$ presented descend tendency. At each dose point of the experiment the SDH activity in $\mathrm{M}$ and $\mathrm{H}$ groups decreased significantly compared with the control groups $(p<0.01)$. Notably, the SDH activities in the $\mathrm{M}$ and $\mathrm{H}$ groups at $90 \mathrm{~d}$ were $38.2 \%$ and $50.9 \%$ lower, respectively $(p<0.01)$.

The CaN activity presented a decreased tendency along with the Mn treatment dose. The CaN activity in the Mn treatment group decreased significantly compared with the corresponding control group $(p<0.05)$. The $\mathrm{CaN}$ activities in the $\mathrm{M}$ and $\mathrm{H}$ groups at $90 \mathrm{~d}$ were $46.7 \%$ and $52.1 \%$ higher, respectively $(p<0.01)$.

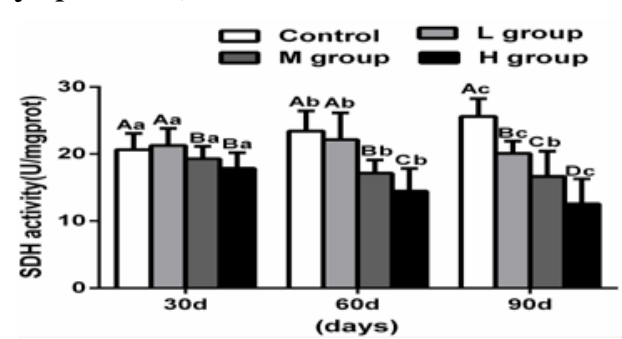

(a) activity of SDH

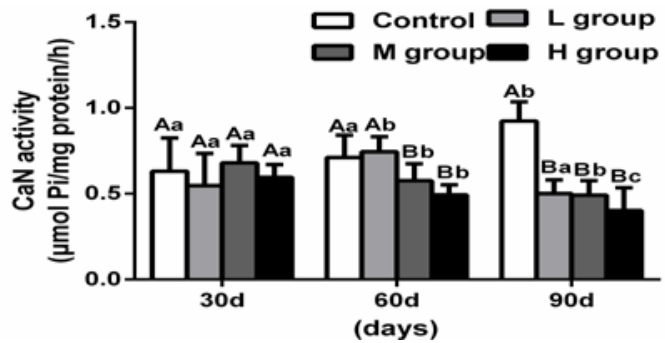

(b) activity of $\mathrm{CaN}$

Fig. 3 Effects of Mn poisoning on variation of the activities of SDH and $\mathrm{CaN}$ i

Apoptotic cells had brown-stain nuclei in TUNEL assay, which showed morphologic changes of condensed and irregular nuclei. There were more apoptotic cells in the diet-Mn group, this phenomenon was obviously in the H group (Fig.4). There were a visible dose-depend fashion in the brain and the apoptotic index in the $\mathrm{M}$ and $\mathrm{H}$ groups increased remarkably than in the other groups $(p<0.01)$. 


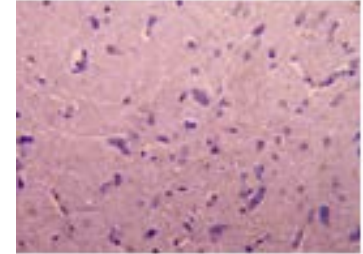

(a) control group

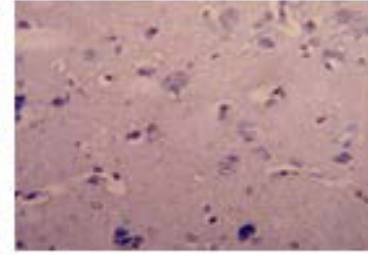

(b) L group

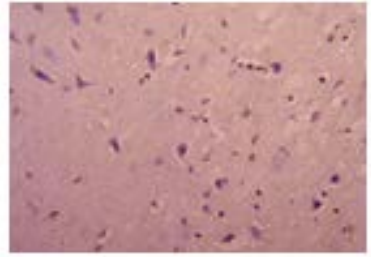

(c) M group.

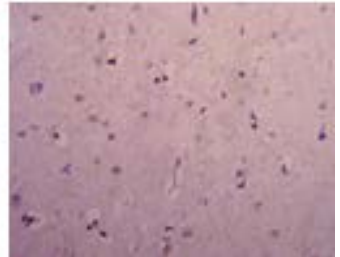

(d) $\mathrm{H}$ group

Fig. 4 TUNEL staining (counterstained with hematoxylin, $\times 400$ ) of cerebral tissue

\section{Discussion}

In this study, a model of Mn poisoning was established to study the effects of dietary Mn on brain damage in cocks. As a transition metal, Mn can also be involved in the production of ROS, and in excess $\mathrm{Mn}$ leads to injury to biological systems [6]. In vitro, $\mathrm{Mn}^{2+}$ ions lead to the production of oxidizing species such as $\mathrm{O}^{2-}$ and $\left.\mathrm{OH}^{-}\right]$. This result was similar to previous reports, illustrating that the inhibition ability of $\mathrm{OH}^{-}$in cock brain in the Mn-diet-fed group was most likely impaired, based on the decreased antioxidant ability.

Mn influx into the retina is faster than its efflux. Milatovic et al [7] eported that a concentration-dependent reduction in ATP was observed at $2 \mathrm{~h}$ following Mn exposure in astrocytes. Tialkens et al [8] postulated that $\mathrm{Mn}$ alters ATP-dependent intracellular $\mathrm{Ca}^{2+}$ dynamics in astrocytes, thereby suppressing $\mathrm{Ca}^{2+}$ wave activity. In certain non-testicular cells, the suppression of ATP production leads to the delay, or inhibition of cell death. Shukla [9] have demonstrated a concentration-dependent effect of $\mathrm{Mn}$ on the brain microsomal $\mathrm{Mg}^{2+}-\mathrm{Na}^{+}, \mathrm{K}^{+}$-ATPase activity. $\mathrm{Mn}^{2+}$ caused an accumulation of succinate within the TCA cycle, consistent with inhibition of SDH.

Apoptosis has been shown to be involved in Mn-induced neurotoxicity. Mn-exposure triggered apoptosis and cell death via the ERK signaling pathway [10]. The present study demonstrated that dietary $\mathrm{Mn}$ induced similar apoptosis in the brain region, as was observed following $\mathrm{Mn}$ administration in previous studies.

\section{Conclusion}

In summary, the oxidative stress and ATPase activities decrease and cell death induced by Mn are important mechanisms in Mn cytotoxicity for bird brain cells. The apoptosis of the neurons induced by excessive Mn are influnced by calcium homeostasis. Therefore, the heavy metal Mn is toxic to brain cells of bird.

\section{Acknowledgement}

In this paper, the research was sponsored by the Scientific Research Fund of Heilongjiang Provincial Education Department(Project No. 12541189).

\section{References}

[1] Burton NC, Guilarte TR. Manganese neurotoxicity: lessons learned from longitudinal studies in nonhuman primates[J]. Environ Health Perspect.2009, 117:325-332

[2] Milatovic D, Yin Z, Gupta RC, et al. Manganese induces oxidative impairment in cultured rat astrocytes[J]. Toxicol Sci, 2007.98: 198--205.

[3] Flora SJS, Megha M, Ashish M. Heavy metal induced oxidative stress and its possible reversal by chelation therapy[J]. Indian. J Med Res, 2008.128: 501--523.

[4] Liu XF. Effects of dietary manganese on $\mathrm{Cu}, \mathrm{Fe}, \mathrm{Zn}, \mathrm{Ca}, \mathrm{IL}-1$, and IL-2 changes of immune Organs in cocks[J]. Biol Trace Elem Res. 2012.148(3): 336-44.

[5] Desole MS, Sciola L, Delogu MR, et al. Role of oxidative stress in the manganese and 
1-methyl-4-(2'-ethylphenyl)-1, 2, 3, 6-tetrahydropyridine-induced apoptosis in PC12 cells[J]. Neurochem. Int, 1997.31(2): 169--176.

[6] Lidon FC, Teixeira MG. Oxygen radical production and control in the chloroplast of Mn-treated rice[J]. Plant Science, 2000.152: 7--15.

[7] Milatovic D, Yin Z, Gupta RC, et al, Manganese induces oxidative impairment in cultured rat astrocytes[J]. Toxicol Sci, 2007.98: 198--205.

[8] Tialkens RB, Zoran MJ, Mohl B, et al. Manganese suppresses ATP-dependent intercellular calcium waves in astrocyte networks through alteration of mitochondrial and endoplasmic reticulum calcium dynamics[J]. Brain Res, 2006. 1113(1): 210--219.

[9] Shukla GS, Malhotra KM, Chandra SV, Effects of manganese on rat brain microsomal $\mathrm{Mg}^{2+}-\mathrm{Na}^{+}-\mathrm{K}^{+}$-ATPase: in vivo and in vitro studies[J]. Environ Res, 1983.32: 212-9.

[10] Yin Z, Aschner JL, dos Santos AP, et al. Mitochondrial-dependent manganese neurotoxicity in rat primary astrocyte cultures[J]. Brain Res, 2008.1203: 1-11. 